\title{
QOS PARAMETERS FOR OBS NETWORK
}

\author{
Sampada D. Samudra', Shilpa P. Gaikwad ${ }^{2}$ \\ ${ }^{1}$ M.Tech Student, Dept. of Electronics, BVUCOEP, Pune, India \\ ${ }^{2}$ Professor, Dept. of Electronics, BVUCOEP, Pune, India
}

\begin{abstract}
In optical circuit switching the high values of blocking probability is resolved by dynamic wavelength routing algorithms with wavelength conversion. The aim of this paper is to study these algorithms. Then the algorithm is selected which gives good results with and without wavelength conversion. The selected algorithm is then checked for other parameters of networking namely throughput, packet delivery ratio, and delay. A comparative study is then carried out for increasing traffic. We try to prove that these algorithms satisfy the criteria of QoS parameters by this comparative study. The results of simulation show that the parameters follow the trend of blocking probability of the selected algorithm.
\end{abstract}

Keywords: optical burst switching, throughput, packet delivery ratio, delay. $* * *$

\section{INTRODUCTION}

Optical networking is an emerging technology for web communication. This technology has the ability to satisfy the need of increasing traffic and the bandwidth required. With the onset of optical networks the issues of high blocking probability and wavelength routing has also emerged. Many researchers have come up with different algorithms to tackle these problems [4][5][6]. These researchers have concluded that wavelength routing is the cause of high blocking probability[7]. To resolve the wavelength routing issue, algorithms have been worked out for dynamic wavelength routing assignment. Some of them have proved to give good results. A novel approach is to calculate the QoS parameters for a proven algorithm. One such algorithm WLCR (Weighted Least-Congestion Routing) by Xiaowen Chu and Bo Li (ref. 1) is chosen for this paper.

The rest of the paper is structured as follows. Section II discusses the flow and reasoning of the selected algorithm. Section III discusses the simulation for the QoS parameters. Section IV analyses the results of the simulation. Section V concludes the paper.

\section{RELATED THEORY}

For an all-optical network routing and wavelength assignment (RWA) plays an important role. The network performance depends on the availability of a free wavelength on the desired route. RWA is classified in two methods: static and dynamic.

In static RWA the route is predetermined without consideration of current traffic load while in dynamic the route is determined considering the current status of traffic. The shortest path routing is a preferred way of routing in static routing. But the performance is limited as there is concentration of traffic on the short routes and the long routes remain unutilized. Hence there is no even distribution of traffic in the network. This results in low link utilization in static routing. Thus static routing is not capable for traffic engineering. Algorithms in dynamic routing have proved to give good blocking performance [2].

While taking into consideration the current traffic status, dynamic routing can be carried out in either distributed or centralized manner. For light-path establishment dynamic routing can be carried out with the help of wavelength conversion.

Solutions for RWA: Researchers have developed several algorithms to solve this problem of RWA. These algorithms have different approaches. The objectives of all these algorithms are reducing the blocking probability of optical networks and enable maximum utilization of links. There are three basic approaches to deal with this problem: fixedrouting, fixed-alternate routing and adaptive routing.

- $\quad$ Fixed routing: the network has fixed route for all source destination pairs.

- $\quad$ Fixed-alternate routing: a routing table is maintained at each node which is an ordered list of routing paths to the destination node. It can be a list of first, second and third shortest paths. At connection request a route is chosen only from this list.

- Adaptive routing: the route is selected on wavelength availability on each link. The actual path depends on the routing policy like shortest path first or least congested path first.

There are three methods for dynamic RWA namely no wavelength conversion, sparse wavelength conversion and full wavelength conversion.

Switching techniques in optical domain are optical circuit switching (OCS), optical burst switching (OBS) and optical packet switching (OPS). OCS depends on lightpath establishment which can be static and dynamic. The lightpath establishment in both the types cannot successfully satisfy the dynamically varying demands of bandwidth of the internet traffic. The internet traffic is more dynamic and bursty in nature. Hence, it becomes difficult to maintain the current state information of the optical network. 
Other approach is optical packet routing, where the packet moves in the optical domain without conversion from optical domain to electronic domain at each node. The header of the packet is processed in the electronic domain and the optical switch is reconfigured as per the contents of the header. This requires fast switching time. Current switching technologies and optical buffering are not matured to support packet switching.

Optical burst switching is designed to attain a balance between OCS and OPS. In this switching network, a data burst is optically switched through the network. The data burst consists of many IP packets. A control burst is priory switched through the network. The control burst configures the switches along the route. An offset-time is set up between the control burst and the data burst to allow processing of the control burst.

\section{PRIOR WORK}

The conventional algorithms do not work well with wavelength conversion as they only consider the free wavelengths and do not account for the route hop length explicitly.

A weighted-shortest-path strategy was proposed by Hsu et al which minimizes the resource cost and maintains a balanced traffic load on among the links [3].

Dewiani, Kuoji Hirata, Khamisi Kalegele, Yoshinobu Higami, Shin-Ya Kobayashi[5] have proposed a backward reservation method to reduce blocking probability for a multi-fiber WDM network. They propose a scheme in which information is collected by signaling of backward reservation on a link whenever a new light-path request arrives. By using the collected information the receiver node selects a combination of wavelength and route. Thus the scheme does not need to exchange link state information. Hence route with many available wavelengths is selected and generation of bottleneck link is avoided. Thus depletion of specific wavelengths along the routes is also avoided. This improves the blocking probability of a multi-fiber WDM network.

Richard Barry and Pierre Humblet [8] introduced a traffic model for circuit switched all-optical network. They use this model to calculate blocking probability of networks with and without wavelength convertors. They study the effect of path length, switch size and interference length on blocking probability. They try to identify important network parameters and investigate the dependence of blocking probability on these parameters.

Asima Nasir [7] has worked on WRONs (Wavelength Routed Optical Network). The simulatoe ns-2 with OWns ( Optical WDM network simulator ) patch is used to perform simulation. A comparative study has been presented by her on the performance parameters like average hop count, average hop delay and link utilization. An exponential traffic source is used for the simulation
Bo Li et al [1] have extended this concept and proposed weight functions which are proportional to no. of free wavelengths and inversely proportional to the route hoplength. The blocking performances are found to be sensitive to the weight functions [1]. In the algorithm, a set of routes have been pre-computed for each source-destination pair. These routes will be re-computed if the network topology is changed. If a light-path connection request comes to a node pair, the system should make a decision to select a route from the pre-computed set of routes, and then assign wavelength(s) to the selected route. The design objective is to carry more traffic on the route while keeping the blocking probability at a low level. A weight value is associated with each candidate route. After calculation of all the weight values a route is selected with maximum weight value to set up the light-path. Once the light-path is setup, for each segment, the wavelength channel with smallest label will be allocated on all the links in that segment [1].

\section{SIMULATION STEPS}

This section investigates the performance of the selected WLCR algorithm over the 14-node NSFNET topology. We have used ns-2.35 as the simulation tool for the algorithm. nOBS Tool for optical communication: ns-2 supports the optical communication with the tool nOBS. Prior to nOBS, ns-2 handled optical communication through OWns (Optical WDM network simulator. OWns was incorporates with the basic characteristics of WDM system like optical switching nodes, virtual topology construction, multi-wavelength links, related switching schemes and algorithms. With the advent of OBS a new tool nOBS was incorporated with ns-2 to deal with optical networking. The optical node and optical link objects in nOBS are the extended version of node and link objects in ns-2. The optical node can perform the functions of ingress and egress nodes. The processes of burstification, routing and scheduling are also performed in the optical node. nOBS incorporates wavelength converters and fiber delay lines(fdl) which perform the task of scheduling.

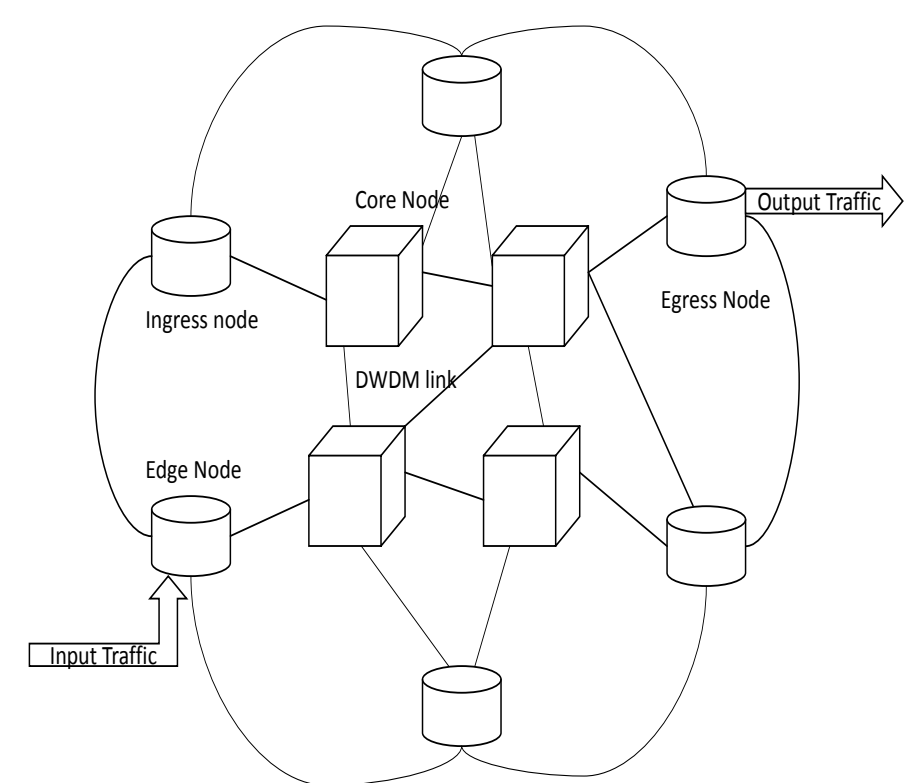

Fig-1 nOBS network 


\subsection{Methodology:}

1. The 14-node NSFNET topology is deployed in the simulation environment.

2. A path is selected from source to destination using shortest path routing.

3. Exponential traffic is used.

4. Alternate path is generated after detecting a block in the shortest path using the WLCR algorithm and a weight function.

5. Traffic is increased in each simulation and data is collected for QoS parameter calculation namely no. of packets sent, no. of packets received, start time and end time.

6. These steps are repeated for all the weight functions.

7. In the simulation, value of every parameter is obtained by conducting 20 replication of the simulation and computing the mean of the results. The simulation compares the performance parameters for three weight functions. The different weight functions are as follows:

$$
\begin{gathered}
w t 1=f / \sqrt{\text { hops }} \\
w t 2=f / h o p s \\
w t 3=1 /\left(\left(1-\frac{f}{w}\right) \sqrt{\text { hops }}\right)
\end{gathered}
$$

where $\mathrm{wt}=$ weight function, $\mathrm{f}=$ free wavelengths, hops $=$ no. of hops for the route.

Table-1: List of Simulation Parameters

\begin{tabular}{|l|l|}
\hline Parameter & Value \\
\hline Topology & NSFNET \\
\hline Traffic Type & TCP \\
\hline Routing Algorithm & WLCR \\
\hline Traffic Generation & Exponential distribution \\
\hline No. of Wavelengths & 8 \\
\hline Delay & $10 \mathrm{~ms}$ \\
\hline
\end{tabular}

\subsection{Algorithm}

Step 1: A weight value is associated for each candidate route. The weight value is calculated by using the above weight functions.

Step 2: The route with maximum weight value is selected to establish a light-path from source node to destination node. If $\mathrm{f}=0$, there are no free wavelengths, the connection request is blocked.

Step 3: After the establishment of light-path, traffic is varied.

Step 4: Values of throughput, delay, pdr is calculated for each traffic value.

Step 5: Steps 2 to 4 are followed for all the weight functions.

\subsection{Qos Parameters Calculation}

The QoS parameters selected are throughput, packet delivery ratio (pdr) and delay.

\section{a. Throughput (Th)}

Throughput is a measure of no. of packets successfully delivered in a network. It is measured in terms of Mbps. The following equation is used to calculate throughput.

$$
T h=\frac{\text { no.of bytes } * 8}{\left(\text { end }_{\text {time }}-\text { start }_{\text {time }}\right)} \text { Mbps }
$$

\section{b. Packet Delivery Ratio (PDR)}

Packet Delivery Ratio (PDR) signifies how many packets reached the destination successfully. The following equation is used to calculate PDR.

$$
P D R=\frac{\text { no.of packets received }}{\text { no.of packets sent }} * 100 \%
$$

c. Delay

Delay is defined as the time taken by the packets to travel from source to destination. Here we calculate end to end delay. The equation used is as follows:

$$
\text { Delay }=\text { end time }- \text { start time }
$$

\section{SIMULATION RESULTS AND DISCUSSION}

To analyze the QoS parameters, the simulation was run for each weight formula. The QoS parameters were calculated for simulation. A database was created for increasing traffic for every weight function. The graphs shown in figure 1, figure 2 and figure 3 are the results of these simulations for the QoS parameters: throughput, pdr and delay respectively. The simulation results are discussed below:

a. Throughput: The calculation of throughput was done by using equation (1). From the graph in figure 1, it can be observed that weight function wt1 gives the best throughput. For all the weight functions the throughput decreases with increasing traffic.

\section{Throughput}

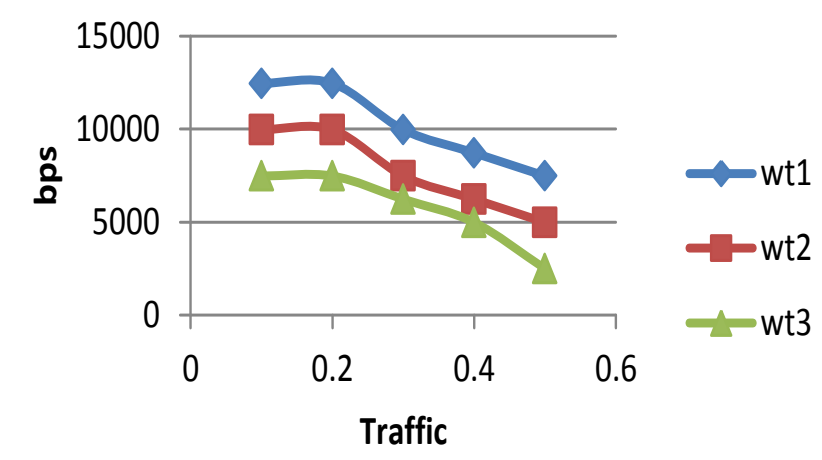

Fig-2: throughput for 3 weight functions. 
b. PDR: Equation (2) is used to calculate the PDR. Figure 2 shows the variation in PDR with increasing traffic. The weight function wt 1 shows the best results for PDR. The value of PDR reduces with increasing traffic.

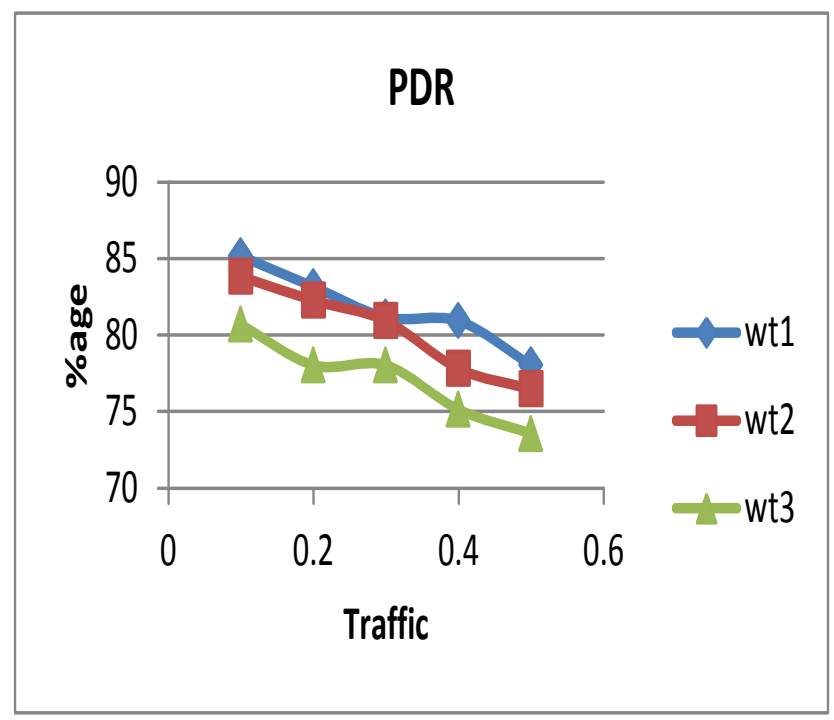

Fig-3 pdr for 3 weight functions

c. Delay: The calculation for delay is done by using equation 3. Figure 3 shows the results of delay with increasing traffic. As the traffic increases the end to end delay also increases. Weight function wt1 shows least delay as compared to other two functions.

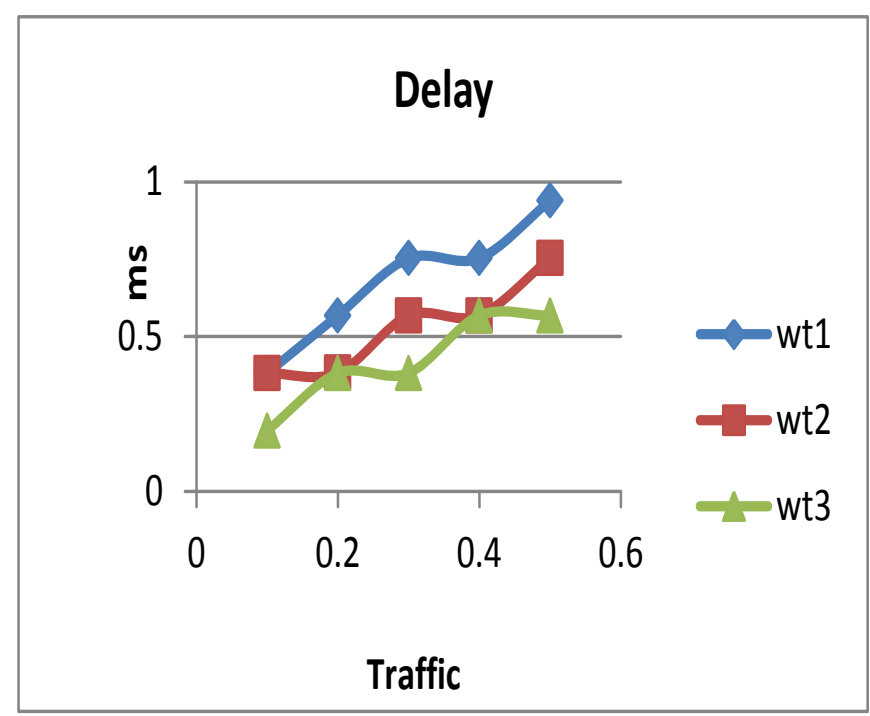

Fig-4: delay for 3 weight functions

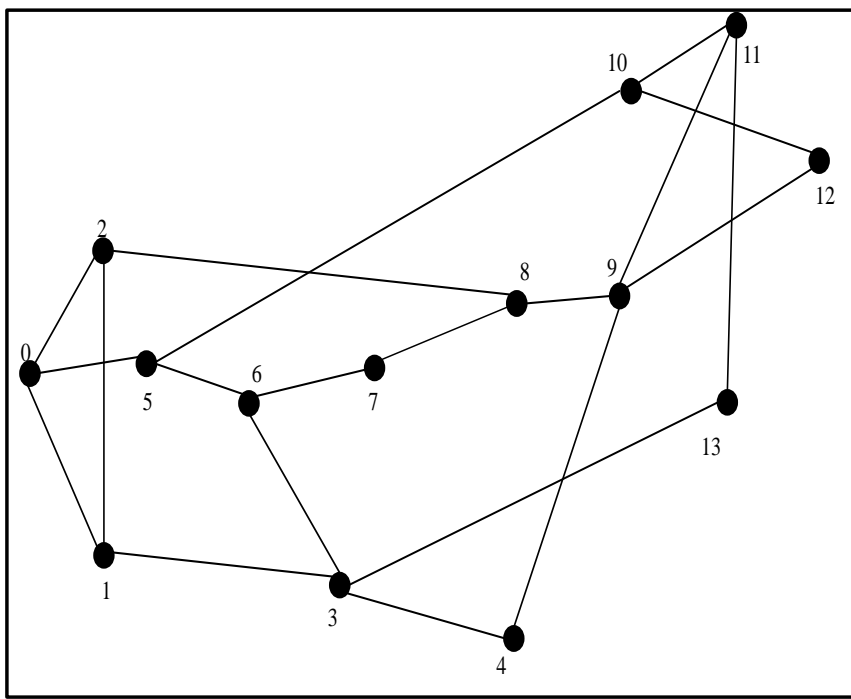

Fig-5: NSFNET topology

\section{CONCLUSION AND FUTURE WORK}

After observing the results of the simulation for all three weight functions, it can be deduced that weight function wt 1 gives optimum values for all the three major QoS parameters. The overall results also suggest that consideration of hop-length and free wavelengths for dynamic routing give good results and a better performance related to QoS parameters. Hence we can conclude that better QoS performance can be attained in an optical network by using the weight functions for dynamic routing. The delay in observed to be inversely related to throughput.

As future work the same approach can be applied to large networks and more no. of wavelengths. The performance of bottlenecks in optical networks with different strengths of wavelengths can be studied.

\section{REFERENCES:}

[1] Xiaowen Chu and Bo Li, "Dynamic Routing and Wavelength Routing in the presence of Wavelength Conversion for All Optical Networks", IEEE/ACM Transaction on Networking, Vol. 13, No. 3, June 2005.

[2] A. Birman, "Computing approximate blocking probability for a class of all-optical networks", IEEE Journal on Selected Areas in Communication, vol. 14, pp. 852-857, June 1996.

[3] C.F. Hsu T.L. Liu and N.F. Huang, "An Adaptive Routing in wavelength-routed networks", SPIE/Kluwer Optical Networks Magazine vol. 3, no. 1, pp 15-24, Jan. 2002.

[4] Aditya Goel, Vitthal Gond, R.K.Singh, A.K.Singh, "Performance Analysis of Continuous Wavelength Optical Burst Switching Networks", International Journal of Engineering, Vol. 3: issue 6, pg. 609-622.

[5] Dewiani, Kouji Hirata, Khamisi Kalegele, Yoshinobu Higami, Shin-ya Kobayashi, "Dynamic Routing and Wavelength Assignment with Backward Reservation in Wavelength Routed 
Multifiber WDM Networks", Journal of Networks, Vol. 7, No. 9, September 2012, pg. $1441-1448$.

[6] Kungmeng Lo, Daryoush Habibi, Quoc Viet Phung, Hoang Nghia Nguyen, "Dynamic Wanelength Routing in All-optical Mesh Networks", 2005 Asia -Pacific Conference on Communication, Perth, Western Australia, 3 - 5 October, 2005.

[7] Hui Zang, Jason P. Jue, Biswanath Mukherjee, “A Review of Routing and Wavelength Assignment Approaches for Wavelength Routed Optical WDM Networks", Optical Networks Magazine, January 2000, pg. $47-60$.

[8] Richard Barry, Pierrie Humblet, "Models of Blocking Probability in All-Optical Networks With and Without Wavelength Changers", IEEE Jounals of Selected Areas in Communications, Vol. 14, No. 5, June 1996.

[9] AsimaNasir, "Comparative Simulation Study to Evaluate Performance Parameters for Multi-hop WRONs by Owns", Journal of Computing, Vol. 2 issue 9, September 2010.

[10] www.isi.edu/nsnam/ns/

\section{BIOGRAPHIES}

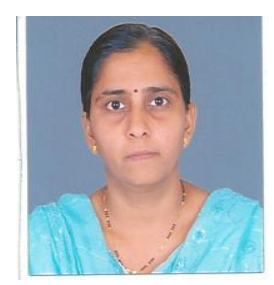

Ms. Sampada Samudra has received B.E. in Electronics and Telecommunication from Pune University, Pune, India. She is pursuing M.Tech. in VLSI from Bharti Vidyapeeth Deemed University, Pune, India

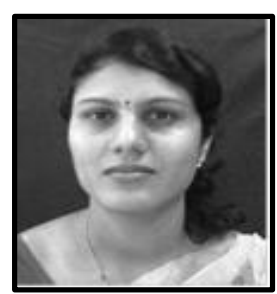

Mrs. Shilpa P. Gaikwad is working as Associate Professor in Dept. of Electronics in BVUCOEP, Pune, India. She is interested in developing and studying new algorithms, architecture and technologies that enhance network efficiency. 